\title{
Presentación del infarto agudo de miocardio con elevación del segmento ST-T en un centro de Ciudad de Buenos Aires durante la pandemia COVID-19
}

\author{
ST-T Segment Elevation Myocardial Infarction in a Center from Buenos \\ Aires city During the COVID-19 Pandemic
}

A. Matías Rodríguez-Granillo1,3, Hernán Pavlovsky², Camila Correa-Sadouet', Camila Gallardo', Axel Vitale', María V. Curotto', Carlos Fernández-Pereira ${ }^{2,3}$, Juan Mieres ${ }^{2,3}$, Ricardo Pérez de la Hoz' ${ }^{1}$ Alfredo E. Rodríguez ${ }^{2,3}$

\section{RESUMEN}

La pandemia COVID-19 presentó un aumento exponencial en el número de internaciones en las unidades de cuidados intensivos alrededor del mundo debido al requerimiento de asistencia ventilatoria y una disminución en la consulta de patologías graves y prevalentes en años previos, en especial de casos graves como el síndrome coronario agudo con elevación del segmento ST-T. En nuestra institución se manifestó el fenómeno con un aumento en los tiempos dolor-primer contacto médico, con presentaciones tardías, y un aumento de las complicaciones intrahospitalarias, incluyendo shock cardiogénico e insuficiencia cardíaca aguda.

Palabras claves: IAMCEST, SCACEST, COVID-19, SARS-COV-2

\section{ABSTRACT}

The COVID-19 pandemic presents an exponential growth in the number of admissions in intensive care units due to the necessity for respiratory support in this patients and a diminish in the consultation for another severe illnesses that were prevalent in years before, such as acute coronary syndromes with ST-T elevation segment. In our institution this phenomena was present with a delayed symptoms-first medical contact, with later presentations and an elevation of complications during hospitalization, including cardiogenic shock and acute heart failure.

Keywords: STEMI, STEACS, COVID-19, SARS-COV-2

Revista Argentina de Cardioangiología Intervencionista 2020;11(2):70-72. https://doi.org/10.30567/RACI/202002/0070-0072

\section{INTRODUCCIÓN}

La alta tasa de contagiosidad del coronavirus-2 (SARSCoV-2) ha dado origen a la pandemia de COVID-19, exponiendo a la salud pública de numerosos países de Asia, Europa y América al colapso debido a la necesidad de soporte ventilatorio en los pacientes complicados, con internaciones prolongadas en la unidad de cuidados intensivos $(\mathrm{UCI})^{1,2}$. Al mismo tiempo, la consulta por otras patologías prevalentes disminuyó de forma significati$\mathrm{va}^{3,4}$. Sin embargo, en la Argentina, donde hasta principios de mayo de 2020 la infección y consecuente mortalidad por COVID-19 son menores a las reportadas en otros países, la disminución de consultas por patologías con elevada morbimortalidad como el infarto de miocardio, sobre todo con elevación del segmento ST-T (STE$\mathrm{MI})$ se desplomó con respecto a años anteriores. Resultados similares se reportan en otros países ${ }^{3,4}$. El objetivo del presente manuscrito es evaluar el impacto inmediato de este hallazgo para prever conductas a fin de mitigar sus consecuencias.

\footnotetext{
1. Servicio de Unidad Coronaria, Sanatorio Otamendi, CABA, Rep. Argentina.

2. Servicio de Cardiología Intervencionista, Sanatorio Otamendi, CABA, Rep. Argentina.

3. Centro de Estudios en Cardiología Intervencionista, CABA, Rep. Argentina. $\triangle$ Correspondencia: Matias Rodriguez Granillo. mrodriguezgranillo@gmail.com Los autores declaran no tener conflictos de intereses.
}

Recibido: 18/05/2020|Aceptado:23/05/2020

\section{MATERIAL Y RESULTADOS}

Desde que la Organización Mundial de la Salud declaró el estado de pandemia por SARS-CoV-2 el 11 de marzo de 2020, muchos países optaron por la cuarentena como método para disminuir la curva de contagio, entre ellos la Argentina, donde comenzó la cuarentena obligatoria el 20 de marzo de 2020 a las 00:00 hs ${ }^{5}$. Desde comienzos de marzo se internaron en nuestra institución 7 pacientes con diagnóstico de STEMI, que fueron incluidos de forma consecutiva en este registro. Se analizaron los datos basales, clínicos y angiográficos de todos los pacientes de forma consecutiva y se compararon con los de los pacientes internados en el año previo por la misma patología. Las características principales de ambos grupos se resumen en la $\mathbf{T a}$ bla 1. En el grupo COVID-19 la edad promedio fue de $70,8 \pm 10,4$ años, el $85,7 \%$ hombres, y con un dolor-primer contacto médico de $552 \pm 300$ minutos. Se realizó ATC primaria en todos los casos, con una tasa de éxito angiográfico del $100 \%$, implantándose 1,8 stents por paciente, y una incidencia de complicaciones del 71,4\%: uno de ellos sufrió un shock cardiogénico, dos pacientes más requirieron diuréticos $\mathrm{EV}$ por presentar KK B al ingreso, uno taponamiento cardíaco y otro sangrado en el sitio de punción. El tiempo de estadía en unidad coronaria fue de $9 \pm 10$ días. Al comparar estos resultados con todos los pacientes con elevación del segmento ST-T en el año 2019 (pre-COVID-19) se evidenciaron diferencias significativas en cuanto al tiempo dolor-primer contacto médico $(\mathrm{p}=0,001)$, sin diferencias en cuanto al tiempo puerta-balón $(\mathrm{p}=0,76)$. Tanto el shock cardiogénico, la fracción de eyección medida por ecocardiografía Doppler color y la incidencia de 


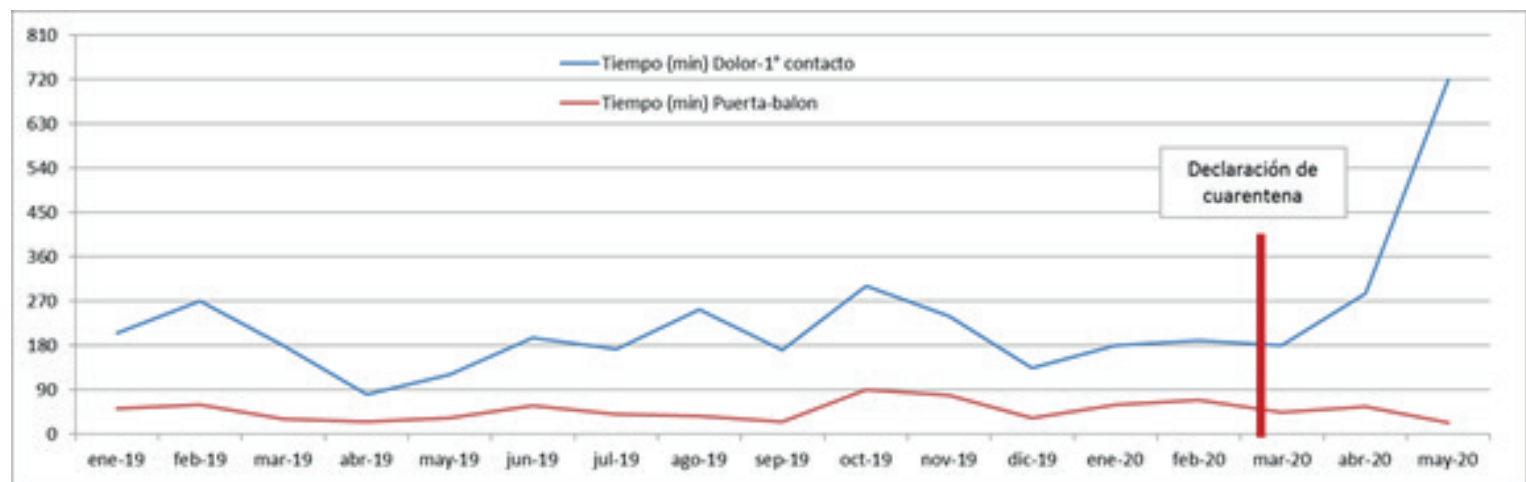

Figura 1. Tiempo de dolor-reperfusión antes y después de la declaración de la pandemia y cuarentena en Argentina. Línea vertical en rojo: fecha de declaración de cuarentena en Argentina.

TABLA 1. Características basales, clínicas y angiográficas de los pacientes con STEMI que ingresaron a unidad cerrada durante la pandemia COVID-19.

\begin{tabular}{|c|c|c|c|c|c|c|c|c|c|c|}
\hline N & Edad & Sexo & $\begin{array}{c}\text { Tiempo dolor-primer } \\
\text { contacto médico } \\
\text { (min) }\end{array}$ & $\begin{array}{c}\text { Tiempo } \\
\text { puerta-ba- } \\
\text { lón (min) }\end{array}$ & $\begin{array}{c}\text { Arteria } \\
\text { culpable }\end{array}$ & $\begin{array}{c}\text { Multiples } \\
\text { vasos }\end{array}$ & Killip K & $\begin{array}{c}\text { Shock } \\
\text { cardiogénico }\end{array}$ & $\begin{array}{c}\text { FEVI } \\
\text { post- ATCp }\end{array}$ & $\begin{array}{c}\text { Tiempo de } \\
\text { internación }\end{array}$ \\
\hline 1 & 57 & Mas & 180 & 45 & DA & No & A & No & Leve & 4 \\
\hline 2 & 78 & Fem & 120 & 60 & DA & Si & B & No & Mod & 4 \\
\hline 3 & 44 & Mas & 360 & 90 & DA & Si & A & No & Leve & 5 \\
\hline 4 & 55 & Mas & 120 & 60 & DA & Si & B & No & Mod & 5 \\
\hline 5 & 63 & Mas & 720 & 15 & DA & Si & D & SI & Severo & 15 \\
\hline 6 & 53 & Mas & 840 & 30 & DA & Si & A & No & Mod & 6 \\
\hline 7 & 61 & Mas & 600 & 15 & DA & Sl & A & No & Mod & 6 \\
\hline
\end{tabular}

sangrado con necesidad de aumentar el tiempo de internación fueron mayores en el grupo COVID-19 en comparación con los pacientes internados previamente, como puede verse en la Tabla 2. En la Figura 1 puede evaluarse el tiempo dolor-primer contacto médico desde enero de 2019 hasta mayo de 2020, marcado con una línea vertical roja el inicio de la pandemia COVID-19.

\section{DISCUSIÓN}

A pesar de su carácter observacional y de confundidores desconocidos, estos hallazgos muestran que el impacto de la cuarentena por COVID-19 en nuestra población ha llevado a un aumento del tiempo de consulta en los casos de infarto, en especial con supradesnivel del segmento ST-T, lo que se traduce en mayor tasa de complicaciones y de tiempos de internación en unidad cerrada, lo que causa un detrimento en los pacientes en particular y en el sistema de salud en general, al aumentar el tiempo de ocupación de camas de alta complejidad. Resultados similares se han reportado en otros países ${ }^{7}$. En una carta al editor de Solomon et al. publicada el 19 de mayo en el New England Journal of Medicine, los autores comunican una disminución en la hospitalización por infarto agudo de miocardio en un hospital de California, Estados Unidos, con respecto a años anteriores; resultados similares se presentaron en el norte de Italia ${ }^{7,8}$. En nuestra experiencia, el inicio de la pandemia se asoció a una disminución en la consulta para luego comenzar una etapa en donde los pacientes consultaron tardíamente, similar a lo publicado recientemente ${ }^{7}$. Esta última etapa no conclu-
TABLA 2. Pacientes con STEMI que ingresaron en Unidad Coronaria en nuestro centro, antes y después de la pandemia COVID-19.

\begin{tabular}{|l|c|c|}
\hline & 2019 (Pre COVID-19) & 2020 (COVID-19) \\
\hline $\mathrm{N}^{\circ}$ & 42 & 7 \\
\hline Edad (años) & $63,5 \pm 13,8$ & $50,6 \pm 10,5$ \\
\hline Sexo masculino & $80,4 \%$ & 85,7 \\
\hline Hipertensión arterial & $63,4 \%$ & $85,7 \%$ \\
\hline Dislipemia & $31,7 \%$ & $14,3 \%$ \\
\hline Diabetes mellitus & $29,3 \%$ & $14,3 \%$ \\
\hline Antecedentes heredofamiliares & $9,8 \%$ & $42,9 \%$ \\
\hline Tabaquistas & $22 \%$ & $14,3 \%$ \\
\hline Enfermedad coronaria previa & $17,1 \%$ & $28,5 \%$ \\
\hline Tiempo dolor-primer contacto & $193 \pm 37$ & $552 \pm 300$ \\
\hline médico (min) & $46 \pm 26$ & $54 \pm 27$ \\
\hline Tiempo puerta-balón (min) & $98 \%$ & $100 \%$ \\
\hline Angioplastia primaria & $150000 \pm 26700$ & $186000 \pm 8270$ \\
\hline Valor pico de Tni (ng/l) & $46,3 \pm 11,8 \%$ & $40,2 \pm 8,5 \%$ \\
\hline FEVI post revascularizacion & $4,7 \%$ & $0,0 \%$ \\
\hline Muerte hospitalaria & $4,7 \%$ & $14,2 \%$ \\
\hline Shock cardiogénico & $4,7 \%$ & $14,2 \%$ \\
\hline Sangrado * & & \\
\hline
\end{tabular}

Tni: troponina ultrasensible; FEVI: fracción de eyección medida por ecocardiografia; KK: clasificación Killip Kimball.

* Sanarado aue hava aumentado el tiempo de internación.

yó en nuestro país. Las consecuencias futuras de estos hallazgos son desconocidas, aunque se infiere de datos previos que la morbilidad podría aumentar, como la insuficiencia cardíaca y la disminución en la calidad de vida de los pacientes afectados. Sugerimos políticas activas para concientizar a la población de estas complicaciones indirectas a causa del COVID-19. 


\section{BIBLIOGRAFÍA}

1. Huang C, Wang Y, LiX, et al. Clinical features of patients infected with 2019 novel coronavirus in Wuhan, China. Lancet 2020;395:497-506.

2. Grasselli G, Zangrillo A, Zanella A, et al. Baseline characteristics and outcomes of 1591 patients infected with SARS-COV-2 admitted to ICUs of the Lombardy Region, Italy. JAMA 2020; doi: 10.1001/jama.2020.5394.

3. Garcia S, AlbaghdadiMS, Meraj PM, et al. Reduction in STsegment elevation cardiac catheterization laboratory activations in the United States during COVID-19 pandemic. J Am Coll Cardiol 2020 April 9 (Epub ahead of print).

4. De Filippo O, D'Ascenzo F, Angelini F, et al. Reduced rate of hospital admissions for ACS during Covid-19 outbreak in northern Italy. NEngl J Med. DOI: 10.1056/NEJMc2009166.

5. WorldHealthOrganization.WHODirector-General'sopeningremarksatthemedia briefing on COVID-19_ 11 March 2020. Available at https://www.who.int/ $\mathrm{dg} /$ speeches/detail/whodirector-general-s-opening-remarks-at-the-mediabriefing-on-covid-19_11-march-2020. Accessed March 12,2020.
6. Cosentino N, Bartorelli NL, Marenzi G, Time to treatment still matters in ST-elevation myocardial infarction: a call to maintain treatment effectiveness during the COVID-19 pandemic, European Heart Journal - Cardiovascular Pharmacotherapy, pvaa054, https://doi.org/10.1093/ehjcvp/pvaa054

7. Solomon MD, MCNulty EJ, Rana JS et al. The Covid-19 Pandemic and the Incidence of Acute Myocardial Infarction. N Enlg J Med. DOI: 10.1056/NEJMc2015630

8. De Filippo O, D'Ascenzo F, Angelini F, et al. Reduced rate ofhospital admissions for ACS during Covid-19 outbreak in northernltaly. NEngl J Med. DOI: 10.1056/NEJMc2009166. 